\title{
Modeling the Dynamics of the Heart Rate Variability by Hidden Markov Models
}

\author{
M Vallverdú, M Palacios, P Caminal \\ Dep. ESAII, Centre for Biomedical Engineering Research, Technical University of Catalonia, \\ Barcelona, Spain
}

\begin{abstract}
A study of the nonlinear dynamics of the Heart Rate Variability (HRV) was done using Hidden Markov Models (HMM). The HRV was obtained from 24-hours Hloter-ECG recordings. The RR series were selected from 6-hour night period of patients with Idiopatic Dilated Cardiomiopathy (IDC) and healthy subjects (NRM). Two groups of patients were considered in the IDC group: HR, patients with high risk of developing sudden cardiac death (SCD); LR, patients without SCD. In the present study, HMMs were identified from the words generated applying Symbolic Dynamics to the RR series. An alphabet of 4 symbols was considered and words of 3 symbols were constructed from the transformed RR series to symbols. Different HMM topologies were analyzed. The logarithm of the observation sequence probability given the model and the maximum probability of the distinct observed words in each state could characterize the HR and LR groups.
\end{abstract}

\section{Introduction}

The Heart Rate Variability (HRV) has been used to study non-invasively the autonomous nervous system [14] and can be used for the diagnostic and prognostic of several cardiac affections. In the last years, several studies have been based on the HRV using methods of the time and frequency domain $[3,4]$. However, these methods give low information about the complex structures involved in the control of cardiovascular system that generates the HRV.

The measurement of the nonlinear properties in HRV signal can help to characterize the intrinsic function that regulates the temporal evolution of the RR series, their nonlinear dynamics. Moreover, several recent studies [2,5] have assessed the clinical usefulness of non-linear measures for high risk stratification based on HRV analysis.

Our work considers the application of Hidden Markov Models (HMM) to the analysis of sequences of words obtained transforming the RR series into symbols of an alphabet, applying symbolic dynamics (SD). The RR series come from two groups of subjects, a group of patients with idiopathic dilated cardiomiopathy (IDC) and a group of healthy subjects (NRM), as control group. Our purpose is to use these statistical models in order to characterize the RR series of IDC patients with high and low risk of suffering sudden cardiac death (SCD). Furthermore, this study is also applied in order to stratify the two groups, healthy subjects and IDC patients.

Two different types of HMM structures are analyzed and different HMM length taken into account; in the same way, different RR series lengths are considered. Two different indices have been obtained from the HMMs able to characterize the different groups of subjects. For the statistical analysis, the nonparametric Mann-Whitney test and a stepwise discriminant function using the living-one-out technique as validation are applied to compare the results between the groups. $\mathrm{p}<0.05$ was considered significant.

\section{Material and methods}

This work is based on the analysis of digital 24-hours Holter-ECG recordings belonging to the IDEAL database [6]. The sampled frequency was $200 \mathrm{~Hz}$. The RR series, distance between two consecutive beats, were obtained automatically from the electrocardiographic signals using a software developed by our group [7]. In order to work with comparable segments, 6-hour segments of the RR series from the night period are considered (from 0:00 to 6:00 am). Three groups of subjects have been taking into account for the analysis: HR, 12 patients with Idiopathic Dilated Cardiomiopathy (IDC) which presented Sudden Cardiac Death (SCD) during the three years after the ECG recording; LR, 32 IDC patients who did not present SCD; NRM, 64 normal subjects considered as control group.

The obtained RR series were transformed to word sequences as result of applying a nonlinear technique, the symbolic dynamics, which eliminates the non-descriptive part of the signal preserving its robust information [5]. These sequences of words have been modeled by HMM with the purpose of characterizing the nonlinear dynamics of the HRV. 


\subsection{Symbolic dynamics}

The symbolic dynamics (SD) is a nonlinear technique which permits to measure the complexity degree of the signal. SD transforms a signal to a pattern, when the samples of the signal are reduced to few symbols, simplifying the study of the dynamical behavior of the signal. A transformation rule based on a simple code was applied to the RR series and then transformed into symbol sequences [5]. An alphabet of 4 symbols $\{1,2,3,4\}$ was considered for our purpose, codifying the $\mathrm{RR}$ series by the following rule:

$$
S_{n}= \begin{cases}1 & (1+a) \mu<R R(n)<\infty \\ 2 & \mu<R R(n) \leq(1+a) \mu \\ 3 & (1-a) \mu<R R(n) \leq \mu \\ 4 & 0<R R(n) \leq(1-a) \mu\end{cases}
$$

where $\mu$ is the mean value of the RR signal and a is a parameter that quantifies the standard deviation of the RR signal $(\mathrm{a}=0.05)$. From these generated series of symbols a sequence of words was constructed containing $\ell=3$ consecutives symbols and without overlapping, obtaining a total of $4^{\ell}=64$ different words (Figure 1).

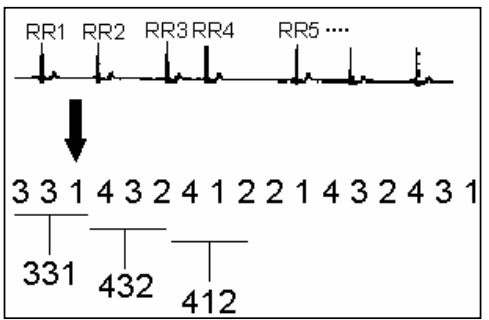

Figure 1. Sequence of words obtained from the RR series.

The word sequences $\left(\mathrm{w}_{\mathrm{ijk}}\right)$ obtained from the RR series have been identified by hidden Markov models.

\subsection{Hidden Markov models}

The HMMs have been widely applied in the speech processing [8] and have been started to be used, during these last years, for the analysis of cardiac arrhythmias in the electrocardiograms [9]. The HMM is a statistical modelling technique that characterizes an observed data sequence by a probability density function which varies according to the state of an underlying Markov chain. To apply this modelling technique one considers the word sequences to be analyzed as the observation sequence of the hidden Markov process, and where the observation is a probabilistic function of the state. The same observation can be generated from other states, with different probabilities. The states are not observable, they are hidden for the observer, since they cannot be directly determined from the observations.

In the present study, HMMs with discrete probability density are assumed since we consider a discrete process where the observation sequences are sequences of words.
The $\mathrm{M}=64$ different words (observed distinct symbols) define the set $\mathrm{V}=\left\{\mathrm{v}_{1}, \mathrm{v}_{2}, \ldots, \mathrm{v}_{\mathrm{M}}\right\}$. In this way, an HMM can characterize the signal (sequences of words or sequences of observations) $\mathrm{O}=\left\{\mathrm{O}_{1}, \mathrm{O}_{2}, \ldots, \mathrm{O}_{\mathrm{T}}\right\}$ by a probability density function which varies by means of the state of the underlying Markov model.

$$
\mathrm{Q}=\left\{\mathrm{q}_{\mathrm{t}} \mid \mathrm{t}=1, \ldots, \mathrm{T}\right\}
$$

where $\mathrm{T}$ is the number of words obtained from the RR signal and $\mathrm{q}_{\mathrm{t}}$ defines the state in the time $\mathrm{t}$.

The number of individual states $\mathrm{N}$ in the model can be defined as $\mathrm{S}=\left\{\mathrm{S}_{1}, \mathrm{~S}_{2}, \ldots, \mathrm{S}_{\mathrm{N}}\right\}$.

The matrix of transition distribution probabilities of the states is defined as $\mathrm{A}=\left\{\mathrm{a}_{\mathrm{ij}}\right\}$, where

$$
\mathrm{a}_{\mathrm{ij}}=\mathrm{P}\left[\mathrm{q}_{\mathrm{t}+1}=\mathrm{S}_{\mathrm{j}} \mid \mathrm{q}_{\mathrm{t}}=\mathrm{S}_{\mathrm{i}}\right] 1 \leq \mathrm{i}, \mathrm{j} \leq \mathrm{N}
$$

The probability distribution of the observed symbols in the state $\mathrm{j}$, is $B=b_{j}(k)$, where

$$
\mathrm{b}_{\mathrm{j}}(\mathrm{k})=\mathrm{P}\left[\mathrm{v}_{\mathrm{k}} \text { in } \mathrm{t} \mid \mathrm{q}_{\mathrm{t}}=\mathrm{S}_{\mathrm{j}}\right] \quad 1 \leq \mathrm{j} \leq \mathrm{N}, \quad 1 \leq \mathrm{k} \leq \mathrm{M}
$$

and the initial distribution probabilities of the states are $\pi=\left\{\pi_{\mathrm{i}}\right\}$, where

$$
\pi_{\mathrm{i}}=\mathrm{P}\left[\mathrm{q}_{1}=\mathrm{S}_{\mathrm{i}}\right] 1 \leq \mathrm{i} \leq \mathrm{N}
$$

In this way, a modeling methodology with HMMs, $\lambda=(\mathrm{A}, \mathrm{B}, \pi)$, needs to specify the parameters $\mathrm{M}$ and $\mathrm{N}$, to estimate the three probability measures $A, B, \pi$ and to fix a model structure. Figure 2 shows one of our selected structures used in this work, a left-right structure with $\mathrm{N}=5$ states.

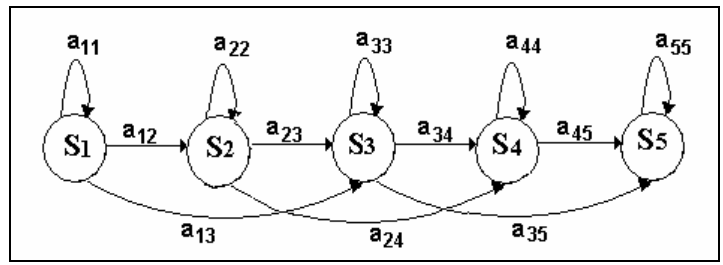

Figure 2. HMM with a 5-state left-right structure.

The HMM parameters (state transition probabilities, output function mean vectors, and covariance matrices) can not be directly estimated from the observation series (the word sequences obtained from the RR series) by a function able to estimate the maximum likelihood, due that the underlying state sequences $\mathrm{S}_{\mathrm{i}}$ which generate the words are unknown. The initial values of the 3-tuple (A, $\mathrm{B}, \pi)$ are used to estimate the model parameters from a set of representative training data by an iterative procedure, this corresponds to the forward-backward algorithm. Viterbi algorithm is used to obtain the state sequence with the highest probability of having generated the word sequences (observation sequence) of a RR series. Finally, the model parameters $(A, B, \pi)$ need to be estimated by maximizing the probability of the observation sequence given the model. We choose $\lambda=(\mathrm{A}, \mathrm{B}, \pi)$ such that $\mathrm{P}(\mathrm{O} / \lambda)$ be locally maximized using 
Baum-Welch iterative procedure.

In the present work, HMMs were studied with two different types of topologies, left-right and ergodic structures, studying models with several number of states $\mathrm{N}=\{5,10,15,20\}$ and windows of $\mathrm{T}=\{120,240,480,960\}$ words along the observation sequences (sequences of words). All parameters $(\mathrm{A}, \mathrm{B}, \pi)$ of the $\lambda$ were randomly initialized. The probability logarithm of the observation sequence given the model $\log [\mathrm{P}(\mathrm{O} / \lambda)]$ and the maximum probability of the observed symbols $\max \left(\mathrm{b}_{\mathrm{j}}(\mathrm{k})\right)$, in each $\mathrm{k}$ states, are the proposed measures obtained from the HMM in order to characterize the analyzed risk groups.

\section{Results}

A statistical analysis was applied to the measures obtained directly from SD: the probability (Prob) of word occurrence $\mathrm{w}_{\mathrm{ijk}}$, the number of words with a probability threshold $(\mathrm{TH})$ higher than the $1 \%$, and the number of words with $\mathrm{TH}<0.1 \%$. Table 1 presents the results of the analysis of those measurements that were obtained with a statistical significant level $\mathrm{p}<0.0005$ and at the same time they were able to discriminate between IDC and NRM groups. The statistical analysis has not presented any accepted statistical significant level $(p<0.05)$ when the groups HR and LR were compared.

Table 1. Mean \pm standard deviation values of the probability of word occurrences obtained from SD. The statistical significant level of these measures is $p<0.0005$.

\begin{tabular}{lllll}
\hline $\mathrm{w}_{\mathrm{ijk}}$ & IDC & NRM & \multicolumn{2}{c}{$(\%)$ Well classified } \\
\hline & $\mu \pm \sigma$ & $\mu \pm \sigma$ & IDC & NRM \\
\hline $\mathrm{w}_{112}$ & $0.026 \pm 0.013$ & $0.043 \pm 0.143$ & 75.0 & 71.9 \\
$\mathrm{w}_{123}$ & $0.004 \pm 0.006$ & $0.012 \pm 0.009$ & 84.0 & 62.5 \\
$\mathrm{w}_{211}$ & $0.025 \pm 0.130$ & $0.041 \pm 0.152$ & 72.7 & 70.3 \\
$\mathrm{w}_{234}$ & $0.003 \pm 0.003$ & $0.008 \pm 0.006$ & 81.8 & 62.5 \\
$\mathrm{w}_{321}$ & $0.004 \pm 0.005$ & $0.012 \pm 0.008$ & 86.4 & 68.8 \\
$\mathrm{w}_{333}$ & $0.124 \pm 0.083$ & $0.065 \pm 0.065$ & 61.4 & 76.6 \\
$\mathrm{w}_{432}$ & $0.003 \pm 0.003$ & $0.007 \pm 0.006$ & 79.5 & 60.9 \\
\hline
\end{tabular}

Table 2. Statistical and discriminant analysis of $\log [\mathrm{P}(\mathrm{O} / \lambda)]$ : $\mathrm{HR}$, high risk of $\mathrm{SCD}$; $\mathrm{LR}$, low risk of SCD.

\begin{tabular}{llll}
\hline HMM left-right & & \multicolumn{2}{l}{ (\%) Well classified } \\
& & & \\
\hline & p-value & HR & LR \\
\hline $\mathrm{HR}_{\mathrm{N}=10}: \mathrm{LR}_{\mathrm{N}=5}$ & 0.002 & 75.0 & 65.6 \\
$\mathrm{HR}_{\mathrm{N}=15}: \mathrm{LR}_{\mathrm{N}=5}$ & 0.003 & 75.0 & 62.5 \\
$\mathrm{HR}_{\mathrm{N}=15}: \mathrm{LR}_{\mathrm{N}=10}$ & 0.020 & 75.0 & 72.0 \\
$\mathrm{HR}_{\mathrm{N}=20}: \mathrm{LR}_{\mathrm{N}=5}$ & 0.004 & 62.5 & 66.7 \\
$\mathrm{HR}_{\mathrm{N}=20}: \mathrm{LR}_{\mathrm{N}=10}$ & 0.028 & 66.7 & 72 \\
\hline $\mathrm{HMM} \mathrm{Ergodic}$ & & & \\
\hline $\mathrm{HR}_{\mathrm{N}=20}: \mathrm{LR}_{\mathrm{N}=10}$ & 0.001 & 75.0 & 65.6 \\
\hline
\end{tabular}

When the sequences of words obtained applying SD were identified by the presented HMM methodology, considering the number of states $\mathrm{N}=\{5,10,15,20\}$ and window lengths of $\mathrm{T}=\{120,240,480,960\}$ words, these have permitted to obtained that HMM with $\mathrm{N}=\{10,15,20\}$ are the number of states that better characterize the HR group and $\mathrm{HMM}$ with $\mathrm{N}=\{5,10\}$ the number of states that better characterize the LR group. The statistical significant levels and the percentage of the well classified patients are presented in Table 2, and obtained from the statistical analysis of $\log [\mathrm{P}(\mathrm{O} / \lambda)]$ values of the identified HMM with the left-right or ergodic structure which better characterize HR and LR groups. The mean values of $\log [\mathrm{P}(\mathrm{O} / \lambda)]$ corresponding to HMMs of Table 2 are presented in Table 3. As it can be observed, the values of $\log [\mathrm{P}(\mathrm{O} / \lambda)]$ in the HR group are higher than in the LR group. Non-significant statistical differences were found when all models presented in Tables 2 and 3 were compared: $\mathrm{HR}_{\mathrm{N}=10}: \mathrm{HR}_{\mathrm{N}=5}, \mathrm{LR}_{\mathrm{N}=10}: \mathrm{LR}_{\mathrm{N}=5} ; \mathrm{HR}_{\mathrm{N}=15}: \mathrm{HR}_{\mathrm{N}=5}$, $\mathrm{LR}_{\mathrm{N}=15}: \mathrm{LR}_{\mathrm{N}=5} ; \quad \mathrm{HR}_{\mathrm{N}=15}: \mathrm{HR}_{\mathrm{N}=10}, \quad \mathrm{LR}_{\mathrm{N}=15}: \mathrm{LR}_{\mathrm{N}=10} ;$ $\mathrm{HR}_{\mathrm{N}=20}: \mathrm{HR}_{\mathrm{N}=5}, \quad \mathrm{LR}_{\mathrm{N}=20}: \mathrm{LR}_{\mathrm{N}=5} ; \quad \mathrm{HR}_{\mathrm{N}=20}: \mathrm{HR}_{\mathrm{N}=10}$, $\mathrm{LR}_{\mathrm{N}=20}: \mathrm{LR}_{\mathrm{N}=10}$. The results of $\log [P(O / \lambda)]$ values corresponding to HMMs with left-right and ergodic structures for $\mathrm{N}=15$ that best characterize to IDC and NRM groups are presented in Table 4. It can be observed that the mean values of $\log [\mathrm{P}(\mathrm{O} / \lambda)]$ corresponding to IDC group are higher than in the NRM group, $\mathrm{p}<0.0005$.

Table 3. Mean \pm standard deviation values of $\log [\mathrm{P}(\mathrm{O} / \lambda)]$ of HMM identification: HR, high risk of SCD; LR, low risk of SCD.

\begin{tabular}{|c|c|c|}
\hline HMM left-right & Groups & $\mu \pm \sigma$ \\
\hline \multirow[t]{2}{*}{$\mathrm{HR}_{\mathrm{N}=10}: \mathrm{LR}_{\mathrm{N}=5}$} & HR & $-924 \pm 229$ \\
\hline & LR & $-1153 \pm 216$ \\
\hline \multirow{2}{*}{$\mathrm{HR}_{\mathrm{N}=15}: \mathrm{LR}_{\mathrm{N}=5}$} & HR & $-934 \pm 224$ \\
\hline & LR & $-1153 \pm 219$ \\
\hline \multirow{2}{*}{$\mathrm{HR}_{\mathrm{N}=15}: \mathrm{LR}_{\mathrm{N}=10}$} & HR & $-934 \pm 224$ \\
\hline & LR & $-1122 \pm 246$ \\
\hline \multirow[t]{2}{*}{$\mathrm{HR}_{\mathrm{N}=20}: \mathrm{LR}_{\mathrm{N}=5}$} & HR & $-953 \pm 205$ \\
\hline & LR & $-1153 \pm 219$ \\
\hline \multirow[t]{2}{*}{$\mathrm{HR}_{\mathrm{N}=20}: \mathrm{LR}_{\mathrm{N}=10}$} & HR & $-953 \pm 205$ \\
\hline & LR & $-1122 \pm 246$ \\
\hline \multicolumn{3}{|l|}{ HMM Ergodic } \\
\hline \multirow{2}{*}{$\mathrm{HR}_{\mathrm{N}=20}: \mathrm{LR}_{\mathrm{N}=10}$} & HR & $-593 \pm 157$ \\
\hline & LR & $-833 \pm 229$ \\
\hline
\end{tabular}

Table 5 presents the optimal probabilities of the $\max \left(b_{\mathrm{j}}\right)$ values (mean \pm standard deviation), which correspond to observed symbols (64 different words), and also the statistical significant level and the percentage of the well classified patients. These results are obtained from ergodic HMMs with $\mathrm{N}=20$ for HR group and $\mathrm{N}=10$ for LR group. It is observed that the mean value of the $\max \left(b_{j}\right)$ is higher in HR group than in LR group, with a statistical significant level $\mathrm{p}<0.05$.

This study has shown that segments of word sequences of length $T=480$ words are enough to characterize the $R R$ of the groups of subjects by measures obtained from HMMs. 
Table 4. Mean \pm standard deviation values, statistical and discriminate analysis of $\log [\mathrm{P}(\mathrm{O} / \lambda)]$. HMM with $\mathrm{N}=15$ states.

\begin{tabular}{lllll}
\hline HMM left-right & $\mu \pm \sigma$ & p-value & $\begin{array}{l}(\%) \\
\text { Well classified }\end{array}$ \\
\hline Groups & IDC & $-1040 \pm 256$ & $<0.0005$ & 75.0 \\
& NRM & $-1282 \pm 248$ & & 71.9 \\
\hline HMM Ergodic & & & \\
\hline Groups & IDC & $-732 \pm 207$ & $<0.0005$ & 75.0 \\
& NRM & $-946 \pm 217$ & & 73.4 \\
\hline
\end{tabular}

Table 5. Mean \pm standard deviation values of the optimal probabilities $\max \left(\mathrm{b}_{\mathrm{j}}\right)$.

\begin{tabular}{|c|c|c|c|c|c|}
\hline \multicolumn{6}{|c|}{ HMM Ergodic $\mathrm{HR}_{\mathrm{N}=20}: \mathrm{LR}_{\mathrm{N}=10}$} \\
\hline \multirow[t]{2}{*}{$\mathrm{W}_{\mathrm{ijk}}$} & \multirow{2}{*}{$\begin{array}{l}\mathrm{HR} \\
\mu \pm \sigma\end{array}$} & \multirow{2}{*}{$\begin{array}{l}\text { LR } \\
\mu \pm \sigma\end{array}$} & \multirow[t]{2}{*}{ p-value } & \multicolumn{2}{|c|}{$\begin{array}{l}(\%) \\
\text { Well classified }\end{array}$} \\
\hline & & & & HR & LR \\
\hline $\mathrm{w}_{223}$ & $0.605 \pm 0.219$ & $0.376 \pm 0.206$ & 0.003 & 75.0 & 71.9 \\
\hline & $0.604 \pm 0.256$ & $0.267 \pm 0.200$ & $<0.0005$ & 75.0 & 87.5 \\
\hline & $0.743 \pm 0.234$ & $0.373 \pm 0.198$ & $<0.0005$ & 66.7 & 87.5 \\
\hline & $0.112 \pm 0.082$ & $0.038 \pm 0.047$ & 0.01 & 66.7 & 81.3 \\
\hline & $0.668 \pm 0.242$ & $0.401 \pm 0.218$ & 0. & 75.0 & 65.6 \\
\hline $\mathrm{W}$ & $0.553 \pm 0.305$ & $0.279 \pm 0.184$ & 0.009 & 66.7 & 75.0 \\
\hline $\mathrm{w}_{334}$ & $0.749 \pm 0.254$ & $0.362 \pm 0$ & $<0.0005$ & 75.0 & 87.5 \\
\hline $\mathrm{w}_{433}$ & $0.672 \pm 0.302$ & $0.363 \pm 0.193$ & 0.001 & 75.0 & 84.4 \\
\hline
\end{tabular}

\section{Discussion and conclusions}

From the results obtained directly of applying SD it can be observed that the HRV of the IDC group presents lower complexity than the NRM group. While the words $\mathrm{W}_{\mathrm{ijk}}$ contain the symbols $\mathrm{S}_{\mathrm{i}}=\{1,4\}$, this means more variability in the RR series, the $\operatorname{Prob}\left(\mathrm{w}_{\mathrm{ijk}}\right)$ value was lower in IDC group than in NRM group. Taking into account the $\mathrm{W}_{\mathrm{ijk}}$ containing only the symbols $\mathrm{S}_{\mathrm{i}}=\{2,3\}$, this means that RR series has lower variability, the $\operatorname{Prob}\left(\mathrm{w}_{\mathrm{ijk}}\right)$ value is higher in the IDC group than in NRM group. All these results presented a statistical significant level $p<0.0005$. However, no significant differences were observed in the statistical analysis of the classification of HR and LR groups.

The identification of the sequences of words by HMM has permitted to find new measures able to describe the complexity of the HRV in HR and LR patients of presenting SCD. These measures have been $\log [\mathrm{P}(\mathrm{O} / \lambda)]$ and $\max \left(\mathrm{b}_{\mathrm{j}}\right)$.

The identified HMMs that best characterize the HR patients group were constructed with a higher number of states $\mathrm{N}$ than the models which characterize the LR group. Moreover, the values of $\log [\mathrm{P}(\mathrm{O} / \lambda)]$ and $\max \left(\mathrm{b}_{\mathrm{j}}\right)$ of the models obtained from group HR were higher than those obtained from LR group. A similar behaviour of these measures was observed when IDC and NRM groups were analyzed, $\mathrm{p}<0.0005$.

It can be concluded that a higher complexity of the HRV is characterized by $\operatorname{lower} \log [\mathrm{P}(\mathrm{O} / \lambda)]$ and $\max \left(\mathrm{b}_{\mathrm{j}}\right)$ values in comparison with a lower HRV complexity where the $\log [\mathrm{P}(\mathrm{O} / \lambda)]$ and $\max \left(\mathrm{b}_{\mathrm{j}}\right)$ values are higher.

Furthermore, the statistical analysis of the measures obtained directly from SD (the probability of word occurrence, the number of words with a probability threshold $\mathrm{TH}$ higher than the $1 \%$, and the number of words with $\mathrm{TH}<0.1 \%$ ) are not able to discriminate between HR and LR groups. However, the measures obtained from HMM are able to characterize both groups.

\section{Acknowledgements}

This work was partially supported by the grant CICYT (TIC 2001-2167-C02-01) and by the research fellowship grant FPU ref. AP2002-4053, both from the Spanish Government.

\section{References}

[1] Pinna GP, Maestri R. Reliability of transfer function estimates in cardiovascular variability analysis. Medical and Biology Engineering and Computing 2001;38:338347.

[2] Tapanainen JM, Seppänen MD, Laukkanen R, Loimaala A, Huikuri HV. Significance of the accuracy of RR interval detection for the analysis of new dynamic measures of heart rate variability. Annals of Noninvasive Electrocardiology 1999;4:10-18.

[3] Lang E, Caminal P, Horváth G, Jané R, Vallverdú M, Slezsák I, Bayes de Luna A. Spectral analysis of heart period variance (HPV) - a tool to stratify risk following myocardial infarction. Journal of Medical Engineering \& Technology 1998;22:248-256.

[4] Task Force of the Europe Society of Cardiology and the Noth American Society of Pacing and Electrophysiology, Heart rate Variability - Standars of measurement, physiological interpretation and clinical use. Circulation 1996;93:1043-1065.

[5] Voss A, Kurths J, Kleiner HJ, Witt A, Wessel N, Saparin P, Osterziel KJ, Schurath R, Dietz R. The application of methods of non-linear dynamics for the improved and predictive recognition of patients threatened by sudden cardiac death. Cardiovascular Research 1996;31:419-433.

[6] IDEAL Database. Intercity Digital ECG Alliance. University of Rochester Medical Center 1997.

[7] Laguna P, Jane R, Caminal P. Automatic detection of wave boundaries in multilead ECG signals: Validation with de CSE database. Computers and Biomedical Research 1994; 27:45-60.

[8] Rabiner LR, Juang BH. A Tutorial on Hidden Markov Models and Selected Applications in Speech Recognition. Proceedings of the IEEE 1989;77:257-286.

[9] Coast DA, Stern RM, Cano GG, Briller SA. An Approach to Cardiac Arrhythmia analysis using hidden Markov models. IEEE transactions on Biomedical Engineering 1990;37:826-836.

Address for correspondence.

Montserrat Vallverdú.

Dep. ESAII, U. Politècnica de Catalunya

Pau Gargallo 5, 08028 Barcelona, Spain. vallverdu@creb.upc.es. 